\title{
Quantifying neuronal morphology A case study of the growth model approach
}

\author{
Andreas Schierwagen and Jaap van Pelt* \\ University of Leipzig, Institute of Computer Science , 04109 Leipzig \\ *Netherlands Institute for Brain Research, 1105 AZ Amsterdam \\ Email: schierwa@informatik.uni-leipzig.de
}

\begin{abstract}
Morphological data on two classes of superior colliculus (SC) neurons have quantitatively been analyzed for dendritic shape parameters. Their frequency distributions were used to optimize the parameters of a dendritic growth model which describes dendritic morphology by a stochastic growth process of segment branching and elongation. Model-generated trees have shape properties closely matching the observed ones. The dendritic trees of each of the two classes of SC neurons are represented by a specific set of growth model parameters, thus achieving morphological data compression.
\end{abstract}

\section{Introduction}

The morphology of nerve cells is assumed to play a decisive role in information processing in the brain. Recent simulation studies with a stochastic model for dendritic growth have shown that the shape properties of various neuron types naturally may arise from peculiarities in neurite growth and branching during dendritic development [1-3]. In this study, morphological data on two classes of neurons from cat superior colliculus (SC) previously published [4-6] have been used to quantify the dendritic anatomy in terms of the parameters of the dendritic growth model.

\section{Material and Methods}

\subsection{Superior colliculus neurons}

Detailed morphological reconstructions were used from neurons stained with HRP [4, 5]. The sample of cells selected for analysis comprised a triplet of neurons each from superficial (SLNs) and deep (DLNs) SC layers (Fig. 1). Their morphology was analyzed quantitatively for a set of shape parameters including degree, centrifugal order of segments and tree-asymmetry index (as a measure of topological tree type). Mean and SD values of the parameters are presented in Table 1 . Their frequency distributions are shown in Fig. 2 (dashed histograms). 


\subsection{Growth model}

The dendritic growth model has recently been presented in [1, 2]. It describes dendritic growth by a stochastic, nonstationary process of segment branching and elongation. In short, the branching probability of a terminal segment per time-bin is given by $p_{i}=C 2^{-S \gamma} B / N n_{i}^{E}$, with $N$ the total number of time bins in the full period of development and $n_{i}$ the actucal number of terminal segments in the tree at time bin $i$. Parameter $B$ denotes the expected number of branching events at an isolated segment in the full period. Parameters $E$ and $S$ determine how strong the branching probability of a terminal segment depends on the actual number of segments and its proximal-distal location in the tree, respectively. Parameter $\gamma$ denotes the centrifugal order of the terminal segment, and $C=n_{i} / \sum_{1}^{n_{i}} 2^{-S \gamma_{i}}$ is a normalization constant, with summation running over all $n_{i}$ terminal segments.

The modular structure of the model facilitates determining optimal parameter values as explained in detail in [2]. Briefly, parameter $S$ is estimated from topological structure via the asymmetry-index, and parameters $B$ and $E$ from the empirical distribution of segment numbers. The segment length parameters (not shown) are optimized following the steps described in [2].
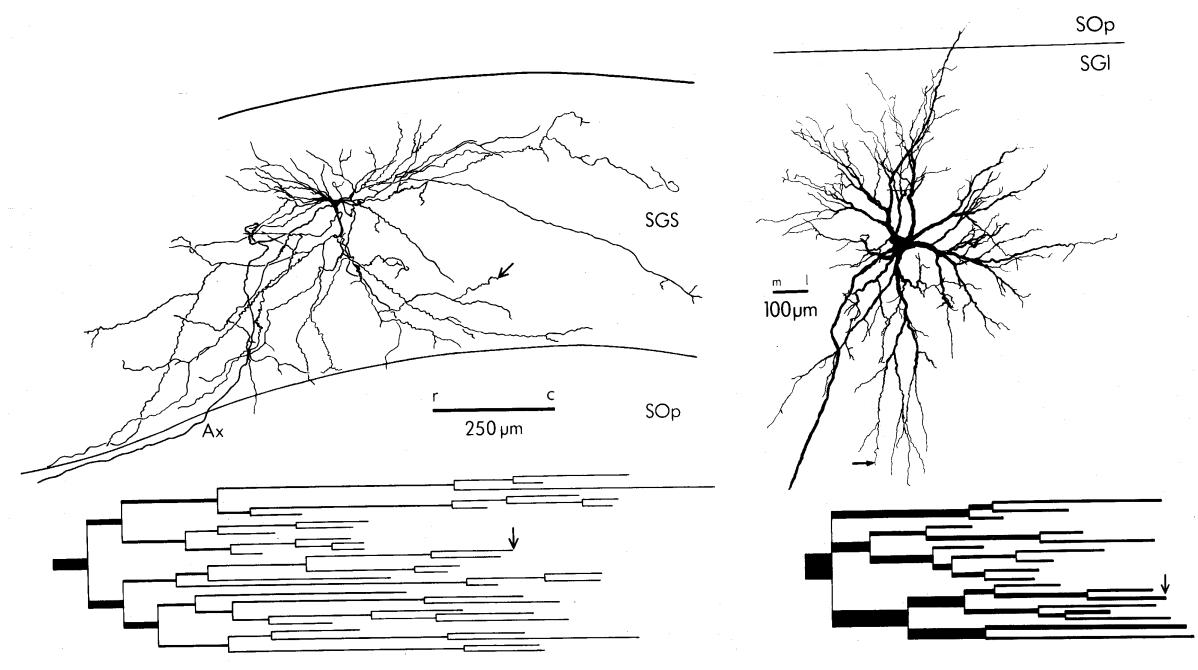

Fig. 1. Projected image of two SC neurons. Left: SLN, right: DLN, each with dendrogram of one dendrite indicated by arrow (below). 


\section{Results}

The frequency distributions of the shape parameters were used to optimize the parameters of the growth model. The parameters of SLNs $(B=4.94, E=0.2, S=0.25$ ) clearly differ from those of DLNs $(B=3.89, E=0.285, S=0.4)$.

The model trees generated with these parameter values have shape properties corresponding closely to the observed ones, as demonstrated in Table 1. In Fig. 2, observed (dashed histograms) and model generated (continuous lines) frequency distributions of shape properties are contrasted.

\section{Cat superficial layer superior colliculus}
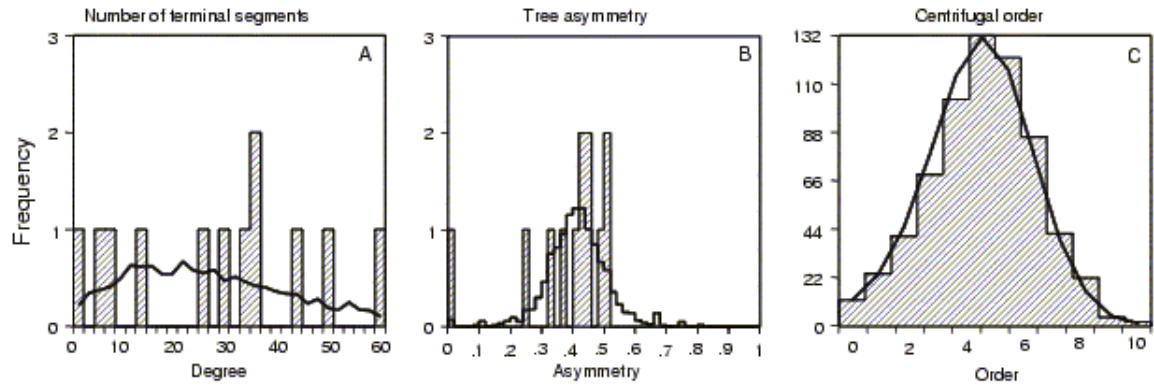

Cat deep layer superior colliculus
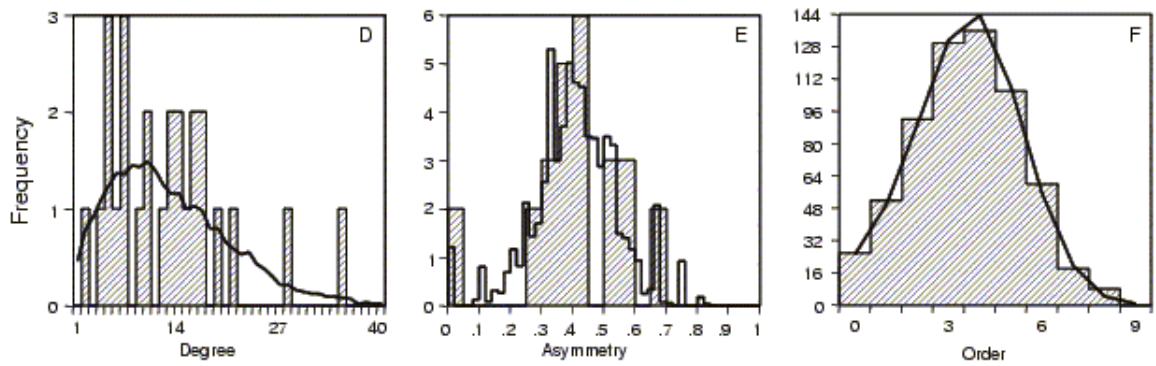

Fig. 2. Frequency distributions of shape parameters of SC neurons (dashed histograms) and model-generated trees (continuous lines) using the optimized parameter values.

\section{Discussion}

The correspondence between frequency distributions of shape parameters derived from reconstructed SC neuron dendrites and model-generated trees indicates that the stochasticity assumptions employed in building the dendritic growth model are successful in explaining the variability of neuronal dendrites. 


\begin{tabular}{|c|c|c|c|c|c|}
\hline Shape variables & \multicolumn{3}{|c|}{ Observations } & \multicolumn{2}{c|}{ Model outcomes } \\
\hline SLNs & $\mathrm{N}_{\text {obs }}$ & Mean & SD & Mean & SD \\
\hline Degree & 26 & 12.58 & 7.46 & 12.49 & 7.39 \\
Tree asymmetry & 26 & 0.41 & 0.15 & 0.41 & 0.14 \\
Centrifugal order & 628 & 3.58 & 1.74 & 3.53 & 1.70 \\
\hline DLNs & $\mathrm{N}_{\text {obs }}$ & Mean & SD & Mean & SD \\
\hline Degree & 12 & 28.3 & 18.1 & 28.6 & 17.7 \\
Tree asymmetry & 12 & 0.39 & 0.14 & 0.42 & 0.1 \\
Centrifugal order & 659 & 5.03 & 2.06 & 4.92 & 2.04 \\
\hline
\end{tabular}

Table 1. Shape properties of observed and modeled dendrites of SC neurons. $N_{o b s}$ denotes the number of experimental manifestations.

Dendritic trees of each of the two classes of SC neurons have been effectively represented by a specific set of the model parameters $B, E$ and $S$, as it has been achieved in previous studies on other neuron types, too [1-3]. Thus, the analysis and comparison of the dendritic shapes of neuron classes during development, maturity and disease become feasible. The growth model also provides a tool for generating sets of random dendritic trees which can be used to explore the functional implications of morphological variations [7].

\section{References}

1. Van Pelt J, Uylings HBM: Natural variability in the geometry of dendritic branching patterns, in: Modeling in the Neurosciences - From Ionic Channels to Neural Networks (Poznanski RR, ed.), Harwood Academic Publishers, Amsterdam, 1999, pp. 79-108.

2. Van Pelt J, Van Ooyen, A, Uylings HBM: Modeling dendritic geometry and the development of nerve connections, in: Computational Neuroscience: Realistic Modeling for Experimentalists (De Schutter E, Cannon RC, eds.), CRC-Press, Boca Raton, FL, 2000, pp. 179-208.

3. Van Pelt, J., A. Schierwagen, H.B.M. Uylings: Modeling dendritic complexity of deep layer superior colliculus neurons. Neurocomputing 38-40: 403-408, 2001.

4. Grantyn R, Ludwig R, Eberhardt W: Neurons of the superficial tectal gray. An intracellular HRP-study on the kitten superior colliculus in vitro. Exp Brain Res 55: 172-176, 1984.

5. Schierwagen A, Grantyn R: Quantitative morphological analysis of deep superior colliculus neurons stained intracellularly with HRP in the cat. J. Hirnforsch., 27: 611-623, 1986.

6. Schierwagen A: Comparative analysis of dendritic geometry and electroanatomy of superior colliculus neurons in the cat. Verh Anat Ges 82 (Anat.Anz.Suppl. 164): 887-890, 1988.

7. Schierwagen A, Claus C: Dendritic morphology and signal delay in superior colliculus neurons. Neurocomputing 38-40: 343-350, 2001. 